\title{
Tiempo de ser diferente, tiempo de ser teacherpreneur y motivar estudiantes
}

\section{It's time to be different, it's time to be a teacherpreneur and motivate students}

\author{
Cristian Abel Huaita Acha \\ Universidad Científica del Sur, Perú
}

Autor por correspondencia: chuaita@gmail.com

Fecha de recepción: 09 de diciembre de 2019 - Fecha de aceptación: 29 de abril de 2020

\section{Resumen}

Uno de los factores determinantes del fracaso y deserción en la educación superior es la falta de motivación, un gran número de estudios ha hecho evidente que muchas instituciones educativas no generan ese vínculo especial entre el disfrute del estudiante en aula y las metodologías utilizadas por el docente. Ante la necesidad de motivar estudiantes a niveles adecuados, la investigación encontró en el "teacherpreneur" o docente emprendedor, a un modelo de docente que respaldado por su metodología y preparación procura que el estudiante forje actitud positiva hacia su futura profesión y se mantenga constantemente motivado. La investigación de tipo experimental y diseño pre experimental buscó demostrar la influencia positiva del teacherpreneur sobre la motivación del estudiante y para tal fin se aplicó en Lima, Perú, en una escuela de negocios, un pre-test y un post-test, antes y después de recibir clases con teacherpreneurs, de tal forma que se compararon los niveles de motivación iniciales con los finales y se obtuvieron resultados categóricos que demostraron el grado significativo de influencia que un teacherpreneur ejerce sobre la motivación de sus estudiantes. Por ello la motivación es el eje impulsor que moviliza toda conducta.

Palabras clave: docente emprendedor; educación superior; emprendimiento; motivación emprendedora; capacidad de motivar.

\begin{abstract}
One of the determining factors of failure and dropout in higher education is the lack of motivation, a large number of studies showed this, making it clear that many educational institutions fail to generate that special link between student enjoyment in the classroom and methodologies used in teaching practice. Given this need to achieve students with suitable levels of motivation, the research found in the "teacherpreneur" or entrepreneurship teacher, a teacher who based on his particular way to teach and preparation looking for that student forges a positive attitude in his future career and maintains constant interest. The experimental research and pre experimental design sought to show how positively a teacherpreneur influences in student motivation and for this purpose a pre-test and post-test was applied to a business school in Lima, Peru; before and after receiving classes with teacherpreneurs, in such a way that the initial levels of motivation were compared against the final ones and categorical results were obtained that demonstrate the significant degree of influence that a teacherpreneur exerts on the motivation of his students. That is why motivation is the driving axis that mobilizes all behavior.
\end{abstract}


Key Words: entrepreneurial teacher; higher education; entrepreneurship; entrepreneurial motivation; ability to motivate.

\section{Introducción}

Una reciente investigación internacional de Milliron y Parnell (2018) halló entre 1500 estudiantes universitarios que los factores que influyen en la deserción y en el fracaso académico se deben a motivos más psicológicos y emocionales que aspectos de tipo vocacional, económico o a la falta de oportunidades. Dichos factores fueron en gran medida la falta de concentración y motivación de los estudiantes, así como también los niveles elevados de ansiedad, carencia de habilidades para el aprendizaje además de la carencia de asesoría. Por tanto, el eje que impulsa y mueve toda conducta y genera cambios tanto a nivel estudiantil y de nuestra propia vida, es la motivación.

Kotler y Armstrong (2008) señalaron que, la motivación también denominada impulso es la que conlleva a una persona a buscar satisfacer una necesidad, cuando la necesidad alcanza un grado suficiente de intensidad, esta se convierte en motivación (p. 177). Asimismo, Hernández (2005) sostuvo que la motivación es innegablemente una parte fundamental de los procesos de aprendizaje y que por tanto influye en el estudiante hasta tal punto que se convierte en uno de los objetivos principales de maestros y de instituciones educativas de nivel superior. Es así que la motivación cumple un rol imperante en el estudiante la cual le permite terminar exitosamente sus metas académicas representando la manera como el estudiante se traza metas y tiene la capacidad lograrlas alcanzar exitosamente, siendo una razón indispensable para guiarse hacia la realización propia logrando elevar su autoestima (La Rosa, 2015).

En el análisis de Milliron y Parnell (2018) se determina que 74\% de universitarios encuestados sentiría un mayor interés y seguridad en sus estudios si acaso se les brindaría asesoría académica, al 70\% le gustaría al menos una asesoría en aula por mes por parte del docente, pero el $20 \%$ solamente lo tiene y un tercio no han tenido contacto con asesor experto alguno en los siete meses últimos, además el $44 \%$ de los encuestados afirmaron que prefieren a "coachings" o mentores expertos para dicho fin, por lo que Ferreyra, Avitabile, Álvarez, Paz y Urzúa (2017) indicaron que, de cada dos personas de 29 años o menos que comienzan estudios en educación superior una no concluye su carrera y casi el $30 \%$ de que se decide por una universidad no la terminarán y más del 30\% desertarán incluso antes del término del primer año.

La Pontificia Universidad Católica del Perú (2018) realizó gran cantidad de estudios con el fin de comprender el porqué del fracaso o deserción estudiantil encontrando que las principales detonantes sobretodo, eran en su mayoría problemas emocionales así como sus dudas vocacionales y las dificultades económicas, y a esto se suma como causa principal justamente la falta de motivación en los estudiantes. Dichos estudios mostraron un preocupante déficit de motivación en el alumno de educación superior. Conseguir que los estudiantes realmente sientan atracción y motivación por los contenidos planteados por los distintos programas, y que esto además sea un impulso para terminar sus carreras satisfactoriamente, es un gran reto.

Al respecto, Sanchez, Ward, Hernández y Flórez (2017) indicaron que, actualmente el sistema educativo tiene como uno de sus grandes desafíos el establecer mecanismos que 
mejor se adecuen con la finalidad de que tanto el emprendimiento, creatividad e innovación sean cruciales a nivel de todos los estamentos educativos, algo que actualmente es una latente preocupación para las entidades de educación y además está generando que varias de ellas se enfoquen en buscar alternativas de cambio diversas, tales como: modificación y adaptación de las mallas de sus currículos hacia modelos educativos que se basen en desarrollar competencias y en la preparación de maestros altamente influyentes en los estudiantes y con capacidades emprendedoras así como espíritu emprendedor. En cambio, Hägg y Schölin (2018) analizaron los fundamentos de las políticas de educación superior que han promovido el espíritu empresarial en Suecia desde mediados de los años noventa. Los autores explican cómo el emprendimiento en la educación superior se rige explícita e implícitamente, por políticas, a través de la creación de nuevas normas en la sociedad.

Para Barbachán, Pareja, Bernardo y Solano (2018) comúnmente se asocia la capacidad de emprendimiento en forma exclusiva a la creación o autogestión de algún negocio, esto constituye un mito, pues el espíritu emprendedor trasciende las capacidades de gestión netamente de empresa y puede irse construyendo a partir de las aulas a edad temprana, propiamente se convierte en una actitud de vida y forma positiva frente a ella. Araque (2015) reforzó esta concepción y afirmó en definitiva que, en efecto, las capacidades emprendedoras se encontraban directamente vinculadas con las actitudes positivas de una persona y sus habilidades para asumir riesgos y con ellos salir exitosos. Asimismo, para Morselli (2018), existe un amplio acuerdo de que el aprendizaje experimental o aprender haciendo, centrado en actividades prácticas con el mundo exterior, ayudan a los estudiantes a desarrollar habilidades y actitudes empresariales. El profesor emprendedor fomenta la toma de riesgos, los estudiantes deben ser aventureros razonables, capaces de pensar y actuar en base a los procesos de toma de decisiones. La educación debe apuntar a desarrollar actitudes como la creatividad, la autoeficacia y el compromiso, pero también debe incluir cierta incertidumbre y ambigüedad.

No obstante, es imposible que se logre desarrollar la mencionada capacidad en los estudiantes sino se toma en cuenta esta propuesta de emprendimiento educativo como el elemento más importante. El docente deja de lado el lugar protagónico de antaño y asume su nuevo rol que es el de un mediador y facilitador en el proceso de aprendizaje, basándose en su experiencia y capacidad debe conseguir en sus estudiantes además de asimilar conocimiento, logre también generarlo y ponerlo en práctica dentro del aula y principalmente en su entorno; y además se sienta satisfecho por realizarlo, quien debe trazarse como fin expulsar esa actitud pasiva hacia el aprendizaje y constantemente ir buscando y mejorando el conocimiento, vale decir, los estudiantes hoy tienen el cometido de enfrentar la vida proactivamente y con un espíritu emprendedor.

El "teacherpreneur" surge como una gran alternativa ante la exigencia de formar estudiantes con alto nivel de motivación, de preparación y con una íntegra formación. El "teacherpreneur" es un docente experimentado, preparado y que apoyándose en la aplicación de una metodología particular que se basa principalmente en la creatividad y en la innovación, pretende que los estudiantes se forjen un espíritu de lucha, actitudes positivas y que mantengan interés constante sobre los contenidos, temas y materias de su carrera (Arruti, 2016).

El teacherpreneur además de destinar tiempo a su labor de enseñanza a sus alumnos, también trata de mejorar la práctica tradicional dentro del aula y las políticas educativas de la comunidad donde se desenvuelve implementando un abanico de nuevas herramientas y de 
estrategias que logren impactar directamente en los estudiantes y en el sector de la educación, un "teacherpreneur" adicionalmente se centra en la búsqueda del cambio del rol del educador usando las herramientas tecnológicas, las virtuales y las del entretenimiento. (Bingham, 2017)

Por consiguiente, la investigación se plantea la interrogante siguiente: ¡el teacherpreneur influye significativamente en la motivación de los estudiantes en una escuela de negocios de Lima? La investigación esperó responder dicha interrogante una vez realizado el trabajo de campo y efectuado el análisis cuantitativo y cualitativo de los resultados.

A esta pregunta se sumó el objetivo general de la investigación, que es determinar la influencia del teacherpreneur en la motivación de estudiantes de una escuela de negocios de Lima, siendo los objetivos específicos, determinar la influencia del teacherpreneur sobre el valor intrínseco, la autoeficacia y la ansiedad de los estudiantes de administración de una escuela de negocios de Lima.

\section{Revisión literaria}

Buckley y Nzembayie (2016) en su investigación que tuvo lugar en Irlanda del Norte y que se denominó "From Vocation to Innovation" y cuyo objetivo fue precisar el nivel de motivación alcanzado por los estudiantes del nivel secundario al implementar estrategias novedosas para la enseñanza que se basan en la creatividad y la innovación, empleando la modalidad virtual y dejando esta labor en manos de "teacherpreneurs", o también llamados docentes emprendedores, nos demostraron que el mundo globalizado en el que se desarrollan actualmente las actividades educativas resulta imprescindible desterrar las metodologías convencionales de enseñanza cambiándolas por metodologías sumamente dinámicas, experimentales e innovadoras las cuales permiten mantener motivados a los estudiantes durante los procesos de aprendizaje.

Otro estudio realizado por Arruti (2016), en España, titulado: "El perfil del teacherpreneur ¿un concepto de moda o realidad?" cuyo objetivo primordial fue el de considerar las características principales que debe tener todo maestro a fin de ser denominado como teacherpreneur, tuvo resultados que resaltaron la importancia de incluir al emprendimiento en la educación moderna sumado a la trascendencia del cambio del rol del profesor de hoy, que se alinea con el espíritu y actitud emprendedora en beneficio de la educación del presente siglo. Además de otras fuentes, la autora basó su investigación en lo planificado por la Comisión Europea para la Educación rumbo al 2020, sobre emprendimiento; en donde se destaca la relación directa y significativa del emprendimiento con la educación. Finalmente el estudio afirmó que la concepción de teacherpreneur más que una simple moda es hoy realidad ya que su aplicación no se limita solo a que los estudiantes puedan proponer algún negocio sino que cataloga al emprendimiento como incluso filosofía de vida que permita al estudiante tener motivación y capacidad suficiente para controlar niveles de stress o ansiedad, valorar las actividades que realiza y el estar seguro de que las hace correctamente y así progresivamente ir alcanzando sus metas.

Strauss (2014) en su estudio titulado "One thing we can learn from Shanghai: how to develop teachers", se planteó determinar por qué en China, provincia de Shanghai, la docencia ha crecido sostenidamente y también como va evolucionando en contraparte de lo que ocurre en otras latitudes, tal es el caso de Estados Unidos donde una cantidad importante de docentes abandonan la docencia a edad temprana y optan por otras profesiones. El estudio demostró que para crear interés y entusiasmo por la enseñanza es vital modificar el perfil 
docente conjuntamente con la dirección que tome en la ruta de conocer técnicas y metodologías innovadoras, logrando la investigación introducir el concepto teacherpreneur como sustento del por qué en China a la docencia se le considera como una atractiva carrera.

También Berry, Byrd y Wieder (2013) en el estudio: "Teacherpreneurs: Innovative teachers who lead but don't leave" consideraron como objetivo el demostrar que los teacherpreneurs podrían ser realmente considerados como un tipo diferente de docentes que pueden lograr un cambio en la vida de sus estudiantes basándose en innovadoras metodologías, contando con habilidades de liderazgo y de motivación ; es así que se expusieron variedad de experiencias recopiladas donde se relataban vivencias dentro y fuera de los salones de clase de teacherpreneurs de diferentes lugares, llegando a la conclusión de que indudablemente el teacherpreneur consigue un efecto inconmensurable e irremplazable en sus alumnos.

Berry (2015) sostuvo en "Teacherpreneurs: Cultivating and scaling up a bold brand of teacher leadership", es momento que los maestros adopten un papel diferente, moderno y no sólo en aulas; ante esta exigencia del sector educativo el teacherpreneur encaja perfectamente. Berry además identificó un número considerable de estudios donde coinciden en la necesidad de destinar mayores recursos para capacitar docentes a fin de que incorporen la filosofía de emprendimiento y sea esta transmitida a los estudiantes, siendo el fin, contribuir con profesionales con actitud y mentalidad positiva a la sociedad, cualidades particulares del teacherpreneur de este siglo. Preliminarmente el mismo autor planteó en "Teacherpreneurs: a bold Brand of teacher leadership for 21st-century teaching and learning", que se justifica los requerimientos de teacherpreneurs del sistema educativo estadounidense debido al gran alcance que tenían hacia sus estudiantes y la comunidad dentro de la aulas y también fuera de ellas, influencia que incluso llega hasta los ambientes productivos donde se desenvuelve, resaltando además las peculiaridades que distinguen a esta clase de docente que tiene condiciones de líder y facilidad en la transmisión de conocimiento y positivismo puro (Berry, 2013).

En México, Soto y Torres (2016) publicaron "Percepciones y expectativas del aprendizaje en jóvenes universitarios", en donde el objetivo era determinar la percepción que tienen los estudiantes referente al proceso de su aprendizaje. En dicho estudio encontraron que la actitud que adopta el docente seguido de su constante actualización en las materias que imparte se consideran como los ejes primordiales para asimilar y crear conocimientos, se propone que el docente adopte un rol como mediador, facilitador, líder y que se convierta en factor de acompañamiento que satisfaga las necesidades que el estudiante tenga durante el desarrollo de las materias y que de acuerdo a los resultados del estudio el profesor no cumple ni dentro ni fuera del aula. Por otro lado Chiecher, Ficco, Paoloni, y García (2016) efectuaron también dentro de México una investigación cuyo objetivo era el análisis de las metas y niveles motivacionales de alumnos de economía considerando el ámbito presencial y también el virtual, logrando encontrar que la motivación, desde los aspectos de autoeficacia, el valor intrínseco que asigna el estudiante a sus labores y la confianza frente a sus niveles de ansiedad, es esencial para los resultados académicos logrados y que de gran manera depende de las estrategias que el docente utilice dentro del aula.

A su turno el mismo año en Cuba otra investigación llamada" La motivación en el rendimiento académico de los estudiantes de medicina", buscó determinar la trascendencia que se obtiene al conseguir en los estudiantes espacios prolongados de motivación a lo largo de toda su carrera. Los autores del estudio buscaron valorar la influencia que tenía la 
motivación en el rendimiento académico de los estudiantes, analizando exhaustivamente el concepto de motivación llegando a concluir que la falta de esta, se relaciona con la autovaloración, la autoestima y la falta de capacidad del estudiante para manejar las preocupaciones y dudas que le genera las actividades y evaluaciones de clase, lo que repercute en el aumento del temprano abandono al inicio de la carrera, sumado a esto las inadecuadas metodologías en aula por parte de los maestros tutores (Jaquinet, Rivero, \& Garnache, 2016)

Según Lens, Matos y Vansteenkiste (2008) existen ciertas teorías, como las de las metas de logro y las de la autodeterminación que contribuyen a explicar del porqué de las diferencias entre el nivel de motivación de los estudiantes de la misma institución y una misma carrera; la investigación está enfocada en la motivación abordándola desde una perspectiva explicada por un indicador de rendimiento, además de interpretarlo en base a niveles o grados de motivación originados en un par de entornos: el docente, familiar y demás entorno educativo en general que influyen en los estudiantes. Según los autores el docente y las estrategias que aplica influyen significativamente en el grado de motivación, incluso se considera al profesor como fuente que motiva al estudiante, alcanzando una posición primordial en el éxito profesional y formación las cuales se logran consiguiendo particulares habilidades a fin de marcar diferencias dentro de sus compañeros de clase y entorno.

Revisada la literatura y considerada la influencia del teacherpreneur en el grado motivacional del estudiante se espera que el presente trabajo de investigación sea un aporte valioso para las entidades de educación de nivel superior, autoridades, estudiantes, docentes, y demás comunidad educativa, así como investigadores y público en general que se interesen por conocer sobre las nuevas propuestas educativas con la finalidad de contribuir en la mejora de la calidad de la educación, puesto que al abordar un tema tratado muy poco hasta ahora, como la filosofía del emprendimiento como herramienta de motivación para estudiantes a través del teacherpreneur, representa un aporte académico frente a la creciente ausencia de motivación en la educación a nivel superior, que en el corto plazo direcciona a los alumnos a desertar y truncar sus carreras.

Por tal motivo esta investigación se justifica en la medida que pretende demostrar que los docentes denominados teacherpreneurs tienen el don de motivar respaldados por su experiencia, capacidad y novedosa metodología a sus estudiantes, de tal manera que los impulsan a conseguir sus objetivos académicos inmediatos y metas personales futuras, no es casualidad que las últimas cifras en el sector evidencian la imperiosa necesidad de hacer un cambio en las prácticas educativas convencionales por metodologías innovadoras y creativas acordes con los tiempos actuales, donde es fundamental el desarrollo de habilidades y competencias, sobre todo habilidades blandas.

En líneas generales se intenta demostrar la hipótesis general de estudio: el teacherpreneur influye significativamente en la motivación de los estudiantes de educación superior de una escuela de negocios de Lima, Perú.

\section{Metodología}

Según Fernández, Hernández y Baptista (2004) se conceptualiza el diseño de investigación como la estrategia o plan preparada con la finalidad de explicar las inquietudes en el proceso de investigación y alcanzar a su vez los objetivos de la misma de manera 
concreta y práctica. La investigación aplicada fue del tipo experimental y con un diseño pre experimental, planteándose la elaboración de un pre test- post test con un grupo experimental. Según Monje (2011) los métodos experimentales realizan cambios controlados en el valor de la variable independiente y observan el efecto en la variable dependiente siendo los más adecuados para poner a prueba hipótesis causales. El enfoque fue mixto pues se consideró el análisis estadístico de los datos recogidos con su respectiva interpretación, se buscó determinar la influencia del teacherpreneur sobre el grado de motivación del estudiante efectuándose para tal fin dos mediciones.

Según Chávez (2007) "La población es el vasto universo del proceso de investigación sobre el que se intenta generalizar resultados mediante la consecución de información y data para su posterior interpretación y análisis". La población estuvo compuesta por estudiantes de la carrera de administración de empresas del grupo de primer ciclo de la primera Escuela de Negocios del Perú, IPAE (Resolución Ministerial 413-2019-MINEDU), La población estuvo conformada por 200 alumnos pertenecientes a ocho secciones de las cuatro sedes de la institución en Lima, dos secciones del primer ciclo por cada sede, de los cuales se tomó una muestra de 100 estudiantes con el objetivo de realizar las mediciones cuantitativas, evaluando a varones y mujeres que tomaron clases con teacherpreneurs, específicamente el curso de comunicación empresarial en la mencionada Escuela de negocios. Malhotra (2004) manifiesta que "el muestreo es la selección o colección de una cantidad determinada de objetos que tengan la categoría de representatividad respecto al total de la población elegida". Para el análisis cualitativo se eligió aleatoriamente del total de las muestras doce elementos. Se aplicaron dos técnicas para la recolección, la entrevista y la encuesta.

\section{Tabla 1}

Tamaño de la muestra sobre una población de 200 estudiantes

POBLACIÓN CANTIDAD

Participantes de primer ciclo quienes tomaron clases con teacherpreneurs 100

$\begin{array}{ll}\text { Total } & 100\end{array}$

El plan a seguir fue realizar dos mediciones, la primera aplicar un pre test inicial de motivación a los estudiantes en la primera semana de inducción al ciclo antes de recibir clases con teacherpreneurs y posteriormente, luego de tres meses de recibir clases con teacherpreneurs de la institución, aplicarles un post test o test de salida a fin de comparar la evolución de sus niveles de motivación.

Los teacherpreneurs, ocho en total, son docentes que han sido capacitados en las incubadoras locales de emprendimiento asociadas a la escuela de negocios, Zegelab y Future Startup Hero, quienes tienen una alianza estratégica con la institución basada en la formación y perfeccionamiento de docentes a quienes se les comparte la filosofía de emprendimiento aplicada a la educación superior.

Hernández, et al. (2004) sostuvieron que la recolección de datos consiste en un proceso mediante el cual se recopilan datos acerca de un hecho, objeto o elemento que esté involucrado con el estudio y para lo cual deben cumplirse tres actividades: se elige el instrumento de investigación que esté a disposición en el entorno académico, se aplica dicho 
instrumento y por último se preparan de los materiales apropiados para el correspondiente análisis.

Dos fueron las técnicas elegidas para recabar los datos, la encuesta y la entrevista para ambos análisis, tanto el cuantitativo como el cualitativo. Las encuestas fueron de tipo presencial y para las entrevistas se consideró las semi - estructuradas debido a que este tipo de entrevista posibilita reformular preguntas debido al alto grado de flexibilidad del contenido.

Para la encuesta se consideró el cuestionario como instrumento. De acuerdo con Bernal (2000) el cuestionario es un conjunto de ítems en formato preguntas que han sido elaboradas con el fin obtener los suficientes datos que conlleven a alcanzar los objetivos de la investigación. Dichos cuestionarios fueron validados previamente a su aplicación a través del "juicio de expertos", mientras que para las entrevistas se utilizó como instrumento la guía de entrevista.

Tabla 2

Calificación del instrumento mediante validación de juicio de expertos

\begin{tabular}{lllll}
\hline $\mathbf{N}^{\mathbf{o}}$ & & Nombre de experto & Especialidad & Calificación instrumento \\
\hline Experto1 & Mag. & Ana Cecilia Napán & Metodóloga & Suficiencia \\
Experto 2 & Dr. & Freddy Luza Castillo & Metodólogo & Suficiencia \\
Experto 3 & Mag. & Jerson Sánchez Ramos & Temático & Suficiencia \\
\hline
\end{tabular}

El primer cuestionario fue adaptado del instrumento original conocido como test MSLQ-SF, en él se desglosa la variable dependiente: motivación del estudiante, en tres dimensiones: valor intrínseco, autoeficacia y ansiedad ante las pruebas, el mencionado test de autoría de Paul Pintrich lanzado hacia 1998 es una herramienta frecuentemente usada dentro del ámbito científico-académico pues mide niveles de motivación y aprendizaje en los estudiantes y a lo largo del tiempo ha venido siendo validado, utilizado y adaptado de forma constante por numerosos investigadores y expertos y aplicado en diversos estudios; es así que para la presente investigación se tomó la versión de veintidós preguntas, esto con el soporte de profesionales académicos quienes validaron una vez más el instrumento. En lo referente al cuestionario para entrevistas este fue diseñado por el investigador y fue también previamente validado.

Bernal (2010) afirma que la validez de un instrumento permite medir en qué grado el cuestionario refleja el dimensionamiento correcto de cada variable.

\section{Fiabilidad del instrumento}

Para asegurar la confiabilidad del instrumento MSLQ-SF, y corroborar la validación del juicio de expertos, se hizo una prueba piloto con la participación de 18 estudiantes de características parecidas a los de la muestra original, con la intención de evaluar el comportamiento del instrumento a la hora de tomar los datos para la consistencia interna, a pesar de como se dijo antes, el mencionado instrumento ha sido validado en el mundo académico en muchas oportunidades. Para este fin se recurrió al uso de una prueba muy recurrente en el campo de la investigación, nos referimos al Alfa de Cronbach. 
Esta prueba del Alfa de Cronbach lo que permite obtener en un trabajo de investigación es la congruencia entre los ítems de un determinado instrumento, así mientras su resultado y valor sea más cercano a uno, será mayor la consistencia del instrumento que se esté evaluando (Vara, 2012).

Para la investigación el Coeficiente de Fiabilidad Alfa de Cronbach obtenido fue de 0,897 en la variable dependiente nivel de motivación de los estudiantes, dicho resultado demuestra que la consistencia interna de la escala del instrumento evaluado es considerada alta, es decir que la estructura de los veintidós ítems de los que está compuesto el cuestionario es considerado bastante adecuado existiendo coherencia en el diseño y estructura del cuestionario.

Tabla 3

Análisis de fiabilidad, variable nivel de motivación del estudiante

\section{ANÁLISIS DE FIABILIDAD}

Coeficiente ALFA de Cronbach =

0,897

Para el análisis respectivo de los datos que se recolectaron se empleó el software estadístico SPSS, mediante el cual se efectuó el análisis descriptivo e inferencial.

Cabe resaltar que la investigación presentó ciertas limitaciones como la demora en la respuesta de los estudiantes encuestados, lo que generó cierta demora también en el procesamiento de los datos. Otra limitante se encontró en el cambio reciente de autoridades de la institución donde se realizó el estudio; ya que en el inicio la investigación se tuvo autorización, pero luego se presentaron algunos inconvenientes con la nueva dirección de la institución. Pero, finalmente, dichas situaciones no representaron riesgo para la realización de la investigación pues fueron manejados y no interfirieron con los objetivos propuestos desde el principio del proyecto. Las acciones a realizar a fin de no tener inconvenientes partieron con la sensibilización de los estudiantes elegidos con la finalidad de agilizar su participación, además como medida complementaria previamente se conversó con los estudiantes seleccionados para las entrevistas, doce en total elegidos aleatoriamente, logrando en ellos una buena disposición para colaborar con la investigación.

La hipótesis general planteada y evaluada fue: El "teacherpreneur" influye significativamente en la motivación de estudiantes de administración de una escuela de negocios de Lima, 2019.

Las hipótesis específicas fueron propuestas en torno a las dimensiones utilizadas para la variable dependiente motivación del estudiante y a continuación se detallan:

El "teacherpreneur" influye significativamente en el valor intrínseco de los estudiantes de administración de una escuela de negocios de Lima, 2019.

El "teacherpreneur" influye significativamente en la autoeficacia de los estudiantes de administración de una escuela de negocios de Lima, 2019.

El "teacherpreneur" influye significativamente en la ansiedad y preocupación ante la dificultad de estudiantes de administración de una escuela de negocios de Lima, 2019. 


\section{Resultados}

Concluida la aplicación de los pre test y post tests y habiendo transcurrido casi cuatro meses desde la toma inicial de muestras hasta las del cierre se presentan a continuación los resultados.

El análisis descriptivo corresponde a la variable de estudio: motivación del estudiante con sus respectivas dimensiones; Los resultados se muestran en las siguientes tablas con los niveles de medición correspondiente para la variable indicada.

A continuación, la siguiente tabla número cuatro detalla los puntajes teóricos, rangos y niveles en donde la variable nivel de motivación alcanzó 103 de puntaje, para las dimensiones los puntajes teóricos se encontraron entre 11 y 40. Se muestra la tabla en donde se detalla la escala valorativa de la motivación del estudiante, dimensionada en valor intrínseco, autoeficacia y ansiedad ante la dificultad.

Tabla 4

Motivación del estudiante, escala valorativa

\begin{tabular}{lccccccc}
\hline \multirow{2}{*}{$\begin{array}{c}\text { VARIABLE Y } \\
\text { DIMENSIONES }\end{array}$} & \multicolumn{2}{c}{ PUNTAJES } & \multicolumn{5}{c}{ NIVELES } \\
\cline { 2 - 8 } & Máximo & Mínimo & $\begin{array}{c}\text { Nada } \\
\text { motivado }\end{array}$ & $\begin{array}{c}\text { Poco } \\
\text { motivado }\end{array}$ & $\begin{array}{c}\text { Moderada } \\
\text { motivación }\end{array}$ & Motivado & $\begin{array}{c}\text { Altamente } \\
\text { motivado }\end{array}$ \\
\hline Motivación del estudiante & 103 & 41 & $41-53$ & $54-66$ & $67-79$ & $80-92$ & $93-103$ \\
Valor intrínseco. & 40 & 11 & $11-16$ & $17-22$ & $23-28$ & $29-34$ & $35-40$ \\
Autoeficacia. & 40 & 16 & $16-20$ & $21-25$ & $26-30$ & $31-35$ & $36-40$ \\
Ansiedad ante la dificultad. & 25 & 12 & $12-14$ & $15-17$ & $18-20$ & $21-23$ & $24-25$ \\
\hline
\end{tabular}

Análisis descriptivo de los resultados, variable motivación y sus dimensiones

\section{Tabla 5}

Niveles de distribución, dimensiones de la motivación de estudiantes I ciclo de administración, escuela de negocios de Lima 2019. Pre test

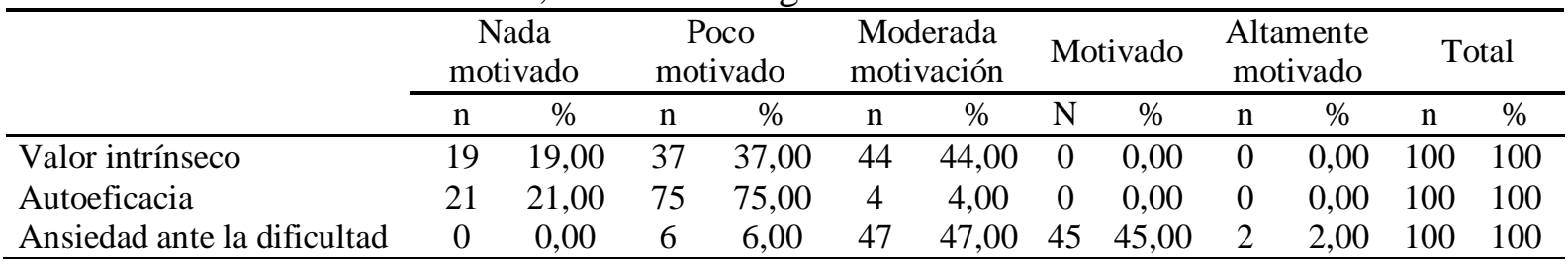

En la tabla 5 se muestran los resultados obtenidos en el pre test; en el que se evidencia que de 100 estudiantes testeados del I ciclo de administración de una escuela de negocios de Lima, para el valor intrínseco, 19 estudiantes que representan el 19\% se muestran nada motivados, 37, es decir el 37\%, muestran poca motivación; mientras que 44 estudiantes, es decir el $44 \%$, muestran una motivación moderada.

En cuanto a la autoeficacia, 21 estudiantes que representan el $21 \%$ no muestran motivación; el 75\% de los estudiantes se encuentran poco motivados y el $4 \%$ de los estudiantes muestran una motivación moderada. 
En la tercera dimensión referente a la ansiedad ante la dificultad, $6 \%$ de los estudiantes muestran poca motivación; $47 \%$ de los estudiantes muestran una motivación moderada; $45 \%$ de los estudiantes se encuentran motivados y un $2 \%$ de los estudiantes se muestran altamente motivados.

\section{Tabla 6}

Niveles de distribución de frecuencias de la variable motivación del estudiante de I ciclo de administración de una escuela de negocios de Lima 2019. Pre test

\begin{tabular}{lcc}
\hline \multicolumn{3}{c}{ MOTIVACIÓN DEL ESTUDIANTE PRE TEST } \\
\hline & Frecuencia & Porcentaje \\
\hline Nada motivado & 21 & 21,0 \\
Poco motivado & 38 & 38,0 \\
Moderada motivación & 41 & 41,0 \\
Motivado & 0 & 0,0 \\
Altamente motivado & 0 & 0,0 \\
Total & 100 & 100,0 \\
\hline
\end{tabular}

En la tabla 6 de frecuencias de la variable motivación observamos que 21 estudiantes o $21 \%$ se encontraron en el nivel nada motivado; el $38 \%$ o 38 estudiantes mostraron poca motivación; mientras que 41 estudiantes o el $41 \%$ mostraron una motivación moderada.

\section{Tabla 7}

Niveles de distribución de las dimensiones de motivación del estudiante de I ciclo de administración de una escuela de negocios de Lima 2019. Post test

\begin{tabular}{lcccccccccccc}
\hline & \multicolumn{2}{c}{$\begin{array}{c}\text { Nada } \\
\text { motivado }\end{array}$} & \multicolumn{2}{c}{ Poco motivado } & \multicolumn{2}{c}{$\begin{array}{c}\text { Moderada } \\
\text { motivación }\end{array}$} & Motivado & \multicolumn{2}{c}{$\begin{array}{c}\text { Altamente } \\
\text { motivado }\end{array}$} & Total \\
\cline { 2 - 13 } & $\mathrm{n}$ & $\%$ & $\mathrm{n}$ & $\%$ & $\mathrm{~N}$ & $\%$ & $\mathrm{n}$ & $\%$ & $\mathrm{n}$ & $\%$ & $\mathrm{n}$ & $\%$ \\
\hline $\begin{array}{l}\text { Valor } \\
\text { intrínseco }\end{array}$ & 0 & 0,00 & 0 & 0,00 & 2 & 2,00 & 47 & 47,00 & 51 & 51,00 & 100 & 100 \\
$\begin{array}{l}\text { Autoeficacia } \\
\text { Ansiedad ante }\end{array}$ & 1 & 1,00 & 16 & 16,00 & 41 & 41,00 & 4 & 4,00 & 38 & 38,00 & 100 & 100 \\
la dificultad & 3 & 3,00 & 32 & 32,00 & 53 & 53,00 & 12 & 12,00 & 0 & 0,00 & 100 & 100 \\
\hline
\end{tabular}

En la tabla 7 se muestran los resultados del post-test; en el cual se evidencia que del total de 100 estudiantes del I ciclo de administración de una escuela de negocios de Lima, para la variable valor intrínseco, 2 estudiantes que representan el $2 \%$ muestran una moderada motivación, 47 estudiantes que representan el 47\% se muestran motivados; mientras que 51 estudiantes o el $51 \%$ muestran un nivel alto de motivación.

En cuanto a la segunda dimensión autoeficacia, 1 estudiante que representa el $1 \%$ no muestra motivacióna; 16 estudiantes que representan el $16 \%$ se encuentran poco motivados; 41 estudiantes que representan el $41 \%$ muestran una motivación moderada; el 4\% (4 alumnos) se muestran motivados y el $38 \%$ (38 alumnos) se encuentran altamente motivados.

En la tercera dimensión referida a la ansiedad ante la dificultad, 3 estudiantes o el 3\% no muestran motivación alguna; 32 estudiantes o el 32\% muestran poca motivación; 53 estudiantes o el $53 \%$ muestran una motivación moderada y el $12 \%$ del total de estudiantes se muestran motivados. 


\section{Tabla 8}

Niveles de distribución de frecuencias de la variable motivación del estudiante de I ciclo de administración de una escuela de negocios de Lima 2019. Post- test

\section{MOTIVACIÓN DEL ESTUDIANTE POST TEST}

Nada motivado

Poco motivado

Moderada motivación

Motivado

Altamente motivado

Total

$\begin{array}{cc}\text { Frecuencia } & \text { Porcentaje } \\ 0 & 0,0 \\ 0 & 0,0 \\ 6 & 6,0 \\ 62 & 62,0 \\ 32 & 32,0 \\ 100 & 100,0\end{array}$

En la tabla 8 referente a motivación de los estudiantes, observamos en el post-test que 6 estudiantes o el 6\% muestran un nivel de motivación moderada; 62 estudiantes o el $62 \%$ se mostraron motivados; mientras que 32 estudiantes o el $32 \%$ se mostraron altamente motivados.

\section{Distribución de la tabla de contingencia, variable motivación del estudiante en el pre test y el post test.}

Tabla 9

Niveles de distribución, comparativo pre test y post test comparativo de la variable motivación de los estudiantes de I ciclo de administración de una escuela de negocios de Lima 2019.

\begin{tabular}{clcccc}
\hline & \multicolumn{2}{c}{ PRE TEST } & \multicolumn{2}{c}{ POST TEST } \\
\cline { 2 - 6 } & & Frecuencia & Porcentaje & Frecuencia & Porcentaje \\
\cline { 2 - 6 } & Nada motivado & 21 & 21,0 & 0 & 0,0 \\
Nivel de motivación & Poco motivado & 38 & 38,0 & 0 & 0,0 \\
del estudiante & Moderada motivación & 41 & 41,0 & 6 & 6,0 \\
& Motivado & 0 & 0,0 & 62 & 62,0 \\
& Altamente motivado & 0 & 0,0 & 32 & 32,0 \\
& Total & 100 & 100,0 & 100 & 100,0 \\
\hline
\end{tabular}

En la tabla 9 se puede apreciar que hubo una diferencia entre los resultados del pre test y el post test en cuanto al nivel de motivación de los estudiantes luego de haber llevado el curso con los teacherpreneurs. Según el pre test $21 \%$, 38\% y $41 \%$ de los estudiantes estaban desmotivados, tenían poca motivación o tenían una motivación moderada respectivamente; y observamos en el post test que esas cifras se redujeron a cero, a excepción de 6 estudiantes que mostraron una motivación moderada. También se aprecia que en el pre test había $0 \%$ de alumnos motivados o altamente motivados; en cambio en el post test las cifras cambiaron, llegando a $62 \%$ y $32 \%$ de estudiantes motivados y altamente motivados respectivamente; lo cual indica que el teacherpreneur si logró un cambio en la motivación de los estudiantes, pero para demostrar el grado de influencia que tuvo se analizó con la estadística inferencial.

\section{Resultados nivel inferencial Prueba de normalidad, bondad de ajuste.}

De acuerdo con Mora (2005), la prueba de bondad de ajuste "tiene una aplicación fundamental cuando para averiguar si la distribución empírica que resulta de cuantificar los 
datos de una muestra se aproxima a la distribución normal, condición que es requerida en numerosas situaciones" (p. 390). Para realizar esta prueba, se recurre al contraste de normalidad de Kolmogorov - Smirnov. Asimismo Mora (2005) señaló como "la prueba más recomendable para testar la normalidad de una muestra, con datos mayores a 50, se basa en medir el ajuste de los datos a una recta probabilística Normal” (p. 395).

La prueba de Kolmogorov-Smirnov se hizo con el software estadístico SPSS 25.

Según Mora (2005) mediante los siguientes pasos:

Paso 1. Plantear la hipótesis alternativa (H1) y la hipótesis nula (Ho):

Ha: Los datos no provienen de una distribución normal

Ho: Los datos provienen de una distribución normal

Paso 2. Seleccionar el nivel de significancia

Para la presente investigación se determinó que: $\alpha=0,05$

Paso 3: Escoger el valor estadístico de prueba

El valor estadístico de prueba considerado para la hipótesis es Kolmogorov Smirnov.

Tabla 10

Prueba de Kolmogorov-Smirnov

\begin{tabular}{|c|c|c|c|c|c|c|}
\hline \multicolumn{7}{|c|}{ PRUEBAS DE NORMALIDAD } \\
\hline & \multicolumn{3}{|c|}{ Kolmogorov-Smirnov ${ }^{\mathrm{a}}$} & \multicolumn{3}{|c|}{ Shapiro-Wilk } \\
\hline & Estadístico & gl & Sig. & Estadístico & gl & Sig. \\
\hline Valor intrínseco - pretest &, 279 & 100 &, 000 &, 781 & 100 &, 000 \\
\hline Autoeficacia - pretest & ,430 & 100 &, 000 & 635 & 100 & ,000 \\
\hline $\begin{array}{l}\text { Ansiedad ante la } \\
\text { dificultad - pretest }\end{array}$ & ,284 & 100 & 000 & ,791 & 100 &, 000 \\
\hline $\begin{array}{l}\text { Motivación del estudiante } \\
\text { Pretest }\end{array}$ & ,262 & 100 &, 000 & ,790 & 100 &, 000 \\
\hline $\begin{array}{l}\text { Valor intrínseco - post } \\
\text { test }\end{array}$ & ,337 & 100 &, 000 & ,692 & 100 &, 000 \\
\hline Autoeficacia -post test & ,281 & 100 &, 000 & ,804 & 100 &, 000 \\
\hline $\begin{array}{l}\text { Ansiedad ante la } \\
\text { dificultad -post test }\end{array}$ & 294 & 100 & ,000 & ,829 & 100 & ,000 \\
\hline $\begin{array}{l}\text { Motivación del estudiante } \\
\text { Postest }\end{array}$ & ,358 & 100 & ,000 & ,728 & 100 & 000 \\
\hline
\end{tabular}

Paso 4: Formulación de regla de decisión

Si alfa $(\mathrm{Sig})>0,05 ;$ Se acepta la hipótesis nula

Si alfa (Sig) < 0,05; Se rechaza la hipótesis nula

Paso 5: Toma de decisión

En la tabla 10 se observa que, el valor calculado de significancia del estadístico de prueba de normalidad resulta en algunas dimensiones menores al valor teórico $\alpha=0,05$; por lo tanto, se rechaza la hipótesis nula y se acepta la hipótesis alterna. Por tanto; según los resultados obtenidos se asume que los datos de la muestra de estudio no provinieron de una distribución normal. Por consiguiente, para el desarrollo de la prueba de hipótesis; se utilizará la prueba no paramétrica de Wilcoxón para distribución no normal, a un nivel de significancia de 0,05 .

\section{Prueba de hipótesis general}

Ha: El "teacherpreneur" influye significativamente en la motivación de los estudiantes de administración de una escuela de negocios de Lima, 2019.

Esta obra se comparte bajo la licencia Creative Common Atribución-No Comercial 4.0 International (CC BY-NC 4.0)

Revista de la Universidad Internacional del Ecuador. URL: https://www.uide.edu.ec/ 
Ho: El "teacherpreneur" no influye significativamente en la motivación de los estudiantes de administración de una escuela de negocios de Lima, 2019.

\section{Nivel de significancia.}

El nivel de significancia teórica es de $\alpha=0,05$, que corresponde a un nivel de confiabilidad de $95 \%$

\section{Regla de decisión.}

Se rechaza Ho cuando la significancia observada "p" de los coeficientes del modelo es menor que $\alpha$.

No rechazar Ho cuando la significancia observada " $p$ " de los coeficientes del modelo es mayor que $\alpha$.

Tabla 11

Prueba de hipótesis entre el pre test y post test de la variable motivación del estudiante

\begin{tabular}{lc}
\hline & \multicolumn{2}{c}{ ESTADÍSTICOS DE PRUEBA } \\
\hline & $\begin{array}{c}\text { Motivación del estudiante Post test - Motivación del } \\
\text { estudiante Pre test }\end{array}$ \\
\cline { 2 - 2 }$Z$ & $-8,708^{\mathrm{b}}$ \\
Sig. asintótica (bilateral) &, 000 \\
\hline a. Prueba de rangos con signo de Wilcoxon \\
b. Se basa en rangos negativos.
\end{tabular}

\section{Decisión estadística}

Como el valor de significancia observada (sig) $\mathrm{p}=, 000$ es menor al valor de la significancia teórica $\alpha=0,05$, nos permite señalar que la diferencia entre el pre test y post test fue estadísticamente significativa, por lo tanto, se rechazó la hipótesis nula y se aceptó la hipótesis alterna, es decir, el "teacherpreneur" influye significativamente en la motivación de los estudiantes de administración de una escuela de negocios de Lima, 2019. De la misma manera se probaron las hipótesis específicas planteadas encontrando diferencia estadísticamente significativa entre el pre test y post test, lo que generó que se rechacen las hipótesis nulas y se acepten las hipótesis alternas.

Respecto a las entrevistas, que se realizaron en el momento en que se efectuó el post test, los resultados arrojaron cifras coincidentes con los hallados en el análisis cuantitativo, $88.9 \%$ de los estudiantes que tomaron clase con teacherpreneurs muestran un grado de motivación alto, $11.1 \%$ un grado moderado y $0 \%$ nada motivado, es decir, los estudiantes corroboraron que los teacherpreneurs son realmente impulsores de motivación en aula, se desprende además la cercanía y confianza que infunden en los estudiantes, lo que los hace diferentes al resto de docentes que aún no lo son.

\section{Discusión}

Un aspecto importante que se debe tener presente es que el emprendimiento no sólo se tangibiliza cuando se forma empresa, eso es algo totalmente alejado de la realidad, puesto que el teacherpreneur o docente emprendedor no comparte ideas de negocio con los estudiantes en aula, lo que hace es compartir ideas y prácticas de vida basadas en un optimismo puro y contagiante que deviene por consecuencia en un constante proceso de motivación. 
Por ello es imposible alcanzar el pleno desarrollo de esta importante capacidad en los estudiantes sino se tiene en cuenta lo más relevante de esta propuesta de emprendimiento aplicado a la educación, que es el maestro y su renovado papel dejando atrás el protagonismo de años pasados tomando nuevos aires que lo conviertan en un mediador y facilitador del aprendizaje, quien apoyándose en su capacidad y experiencia le toca la labor de lograr que el estudiante no reciba únicamente aprendizaje y conocimiento, sino que lo cree y lo practique a todo nivel social, que además sienta satisfacción de hacerlo, y ponerse como objetivo desechar las actitudes pasivas de aprendizaje conduciéndose a una sostenida búsqueda y mejora del conocimiento. La pasividad disminuye la motivación y la curiosidad de los estudiantes, por ello cambia el docente cambia el estudiante, o sea, los estudiantes tienen la responsabilidad de enfrentar la vida con actitud dinámica retroalimentativa y espíritu emprendedor. Como lo señala $f(B$ Bingham (2017), los maestros emprendedores promueven ideas audaces, experiencias y mejores prácticas que se encuentran en núcleo de la profesión.

El análisis de los resultados del post test combinado con las respuestas de las entrevistas de los participantes confirman la hipótesis planteada y revelan que, el teacherpreneur influye significativamente en la motivación de estudiantes de administración de una escuela de negocios de Lima, 2019, estos resultados se interpretan dado que este tipo de docente en aula, no tiene aún nombre propio, más bien el estudiante suele asociarlo con alguna cualidad, talento, característica o temperamento propio de cada docente, significa que el concepto particular de teacherpreneur es más el estilo de docencia que hace del estudiante una persona motivada, esto sucede probablemente porque el docente emprendedor brinda oportunidades de elección y control, sobre todo los estudiantes generalmente se motivan o dirigen su comportamiento hacia actividades que valoran y en las que tienen alguna expectativa de éxito. Asimismo, se percibe a la luz de los resultados que las investigaciones previas en otras latitudes tienen un alto grado de coincidencia con los resultados obtenidos en la presente investigación, apoyando la teoría o filosofía sobre el emprendimiento aplicado al desarrollo educativo en aras de motivar e incentivar el aprendizaje en aula. Lo señalado concuerda con el estudio de Berry (2015) en su artículo titulado: Maestros emprendedores, cultivando y ampliando una marca audaz de liderazgo docente, donde el autor sostuvo que muchos maestros, tal vez más de un millón, podrían liderar como maestros emprendedores si tuvieran una preparación adecuada, apoyo y estructuras de trabajo. Berry describe tres estrategias de alto apalancamiento para ampliar el emprendimiento docente: elevar y publicitar el papel de los líderes docentes, rediseñar la evaluación docente y los sistemas de pago, y preparar y socializar formalmente a los nuevos docentes como docentes emprendedores.

Se confirma también las hipótesis específicas planteadas: El "teacherpreneur" influye significativamente sobre el valor intrínseco, la autoeficacia y la ansiedad de los estudiantes de administración de una escuela de negocios de Lima. Lo que equivale, primero, para construir una motivación intrínseca, el teacherpreneur construye un clima de comprensión y confianza, ayuda a superar desafíos, permite que el estudiante se convierta en competente, se involucre e interactúe con otras personas, incorpora comportamientos instructivos que motivan a los estudiantes. Segundo, que los estudiantes con altas posibilidades de autoeficacia poseen mayor motivación académica, desarrollar el pensamiento crítico para saber tomar decisiones, logran mejores resultados, desarrollan la capacidad de autorregular eficazmente su aprendizaje y por último la ansiedad leve o moderada cumple una función útil, favorece al estudiante puesto que genera un estado de alerta y de tensión que contribuye y mejora el rendimiento. 
Realizada la investigación debemos reconocer además que, para efectos de los resultados obtenidos, la cercanía a la población de estudiantes objeto de estudio, ha contribuido en la consecución de la información dentro del tiempo planificado, es propicio reconocer también que el tamaño de la población elegida influye en la consecución de dichos resultados y su posterior análisis. No obstante, se considera que este es un buen inicio para que otras investigaciones puedan seguir explorando acerca de las nuevas formas de llegar a motivar a los estudiantes de educación superior, de tal manera que se pueda confirmar que la filosofía del emprendimiento no se encuentra divorciada con la práctica docente, tal como se afirma al inicio del presente artículo en los estudios de Berry (2015) y Arruti (2016), quienes coinciden en que el aporte del teacherpreneur termina siendo vital y valorado por los estudiantes, influyendo en las decisiones que los estudiantes toman, de seguir adelante con sus carreras, además de corroborar que este tipo de docente no se trata sólo de una moda sino que se trata de una innegable realidad.

\section{Conclusiones}

El teacherpreneur influye significativamente en la motivación de estudiantes de educación superior. Es preciso mencionar, además, investigaciones como esta y otras relacionadas, nos llevan a reflexionar sobre el momento trascendente que atraviesa el docente de educación superior, vinculado a la gran responsabilidad de tener que cambiar las viejas prácticas en aula por nuevas y mejores formas de mayor dinamismo, de tal manera que cautiven al estudiante y lo lleven a conseguir sus objetivos.

Como consecuencia de los resultados expuestos se afirma también que los estudiantes son influenciados significativamente por el teacherpreneur en su valor intrínseco, es decir, contribuye este con la capacidad de autovaloración que tiene el estudiante sobre las actividades que desarrolla como parte de su carrera profesional, además de generar en ellos un grado de importancia relevante que alimenta su autoeficacia y disminuye considerablemente sus niveles de ansiedad frente a las dificultades que encuentra a lo largo de su desempeño académico.

Este modelo beneficia la educación, logrando en los estudiantes ser los constructores de sus proyectos y de sus ideales. Asimismo, ayuda a los estudiantes en sus capacidades y habilidades sociales como la empatía, liderazgo, temperamento, influyendo además positivamente en sus comportamientos, personalidades y rendimiento académico. El mensaje es directo para los docentes, es hora y tiempo para llegar a ser un teacherpreneur, motivar y ser visto como modelos de persona a seguir.

Se considera además que este es un buen inicio para que otras investigaciones puedan seguir explorando acerca de las nuevas formas de llegar a motivar a los estudiantes de educación superior con el fin de poder potenciar los nuevos aprendizajes que se van asimilando.

\section{Bibliografía}

Araque, W. (2015). Emprendimiento en Ecuador. Ekos Negocios, 72-78. Recuperado de http://portal.uasb.edu.ec/UserFiles/385/File/Emprendimiento\%20en\%20Ecuador.pdf.

Arruti, A. (2016). El desarrollo del perfil del" teacherpreneur" o profesor-emprendedor en el currículum del grado de Educación Primaria: ¿un concepto de moda o una realidad? 
Contextos educativos. Revista de educación, (19), 177-194. Recuperado de https://publicaciones.unirioja.es/ojs/index.php/contextos/article/view/2770.

Barbachán, E. A., Pareja, L., Bernardo, M., \& Solano, J. (2018). Preferencias cerebrales, capacidad emprendedora y personalidad eficaz. Una relación necesaria para los estudiantes universitarios de Perú. Investigación y postgrado, 33(2), 5-5. Recuperado de https://dialnet.unirioja.es/servlet/articulo?codigo $=6736279$.

Bernal, C. (2000). Metodología de la Investigación para Administración y Economía. Bogotá: Editorial Prentice Hall.

Bernal, C. (2010 ). Metodología de la investigación. Bogotá: Pearson Educaciòn (Tercera ed.).

Berry, B. (2013). Teacherpreneurs: A bold brand of teacher leadership for 21st-century teaching and learning. Science, 340(6130), 309-310.

Berry, B. (2015). Teacherpreneurs: Cultivating and scaling up a bold brand of teacher leadership . The New Educator, 11(2), 146-160.

Berry, B., Byrd, A., \& Wieder, A. (2013). Teacherpreneurs: Innovative teachers who lead but don't leave. John Wiley \& Sons. Editorial Reviews.

Bingham, C. S. (2017). What is a Teacherpreneur? Ask Sarah Henchey. Carolina del Sur. Recuperado de https://gardner-

webb.edu/Assets/gardnerwebb/academics/cild/teacherpreneruship-notes1.pdf: Associate Professor of Education, Gardner-Webb University.

Buckley, A. P., \& Nzembayie, K. F. (2016). Teacherpreneurs: From Vocation to Innovation. . Conferencia Internacional sobre Innovación y Emprendimiento. (pág. 36 Volumen: 4to). Canadá: Reseachgate.

Chávez, N. (2007). Introducción a la investigación educativa. Maracaibo: Gráfica González.

Chiecher, A. C., Ficco, C. R., Paoloni, P. V., \& García, G. A. (2016). ¿Qué mueve a los estudiantes exitosos? Metas y motivaciones de universitarios en las modalidades presencial y distancia.,. Revista Observatorio, 2(2), 301-326. Recuperado de https://hal.archives-ouvertes.fr/hal-01560453/document.

Ferreyra, M. M., Avitabile, C., Álvarez, J. B., Paz, F. H., \& Urzúa, S. (2017). Momento decisivo: la educación superior en América Latina y el Caribe. Washington, DC:: Resumen. Banco Mundial. Licencia: Creative Commons Attributi. Recuperado de https://openknowledge.worldbank.org/bitstream/handle/10986/26489/211014ovSP.pd f? sequence $=5 \&$ is Allowed $=\mathrm{y}$.

Hägg, G., \& Schölin, T. (2018). The policy influence on the development of entrepreneurship in higher education: A Swedish perspective. Educación y Entrenamiento. Emerald Group Publishing, https://doi.org/10.1108/ET-07-2017-01 Vol. 60 No. 7/8, pp. 656673. 
Hernández, A. P. (2005). La motivación en los estudiantes universitarios. Revista Electrónica Actualidades Investigativas en Educación, 5(2), 1-13.h. Recuperado de https://www.redalyc.org/pdf/447/44750219.pdf.

Hernández, R., Fernández, C., \& Baptista, P. (2004). Metodología de la investigación . México: ( $3^{\circ}$ ed.). McGraw-Hill interamericana.

Jaquinet, M., Rivero, M. L., \& Garnache, A. Z. (2016). La motivación en el rendimiento académico de los estudiantes de medicina. Revista Médica Electrónica, 38(6), 910915. Recuperado de Recuperado de http://scielo.sld.cu/scielo.php?script=sci_abstract\&pid=S1684$18242016000600013 \& \operatorname{lng}=$ es\&nrm=iso.

Kotler, P., \& Armstrong, G. (2008). Principios de marketing. Madrid: Pearson Educación.

La Rosa, C. (2015). Perspectiva de la motivación en estudiantes universitarios para el logro de metas académica. Ciencias de la Educación, 26(46), 197-213. Recuperado de http://servicio.bc.uc.edu.ve/educacion/revista/46/art16.pdf.

Lens, W., Matos, L., \& Vansteenkiste, M. (2008). El profesor como fuente de motivación de los estudiantes: Hablando del qué y del porqué del aprendizaje de los estudiantes. $R I D U, 4(1), 2$. Recuperado de https://revistas.upc.edu.pe/index.php/docencia/article/view/9/158.

Malhotra, N. K. (2004). Investigación de mercados. México: Pearson educación.

Milliron, M., \& Parnell, A. (25 de junio de 2018). Students perspectives on graduation barriers and advising support . Obtenido de Civitas Learning: https://go.civitaslearning.com/1/481481/2018-06-25/rxhyv

Monje, C. A. (2011). Metodología de la investigación cuantitativa y cualitativa. Guía didáctica. Colombia: Universidad Surcolombiana. Recuperado de https://www.coursehero.com/file/19096448/Gu\%C3\%ADa-did\%C3\%A1cticaMetodolog\%C3\%ADa-de-la-investigaci\%C3\%B3n-Monje-Carlos-Arturo/.

Mora, A. I. (2005). Guía para elaborar una propuesta de investigación. Revista Educación, 29(2), 67-97. Recuperado de https://www.redalyc.org/pdf/440/44029206.pdf.

Morselli, D. (2018). How do Italian vocational teachers educate for a sense of initiative and entrepreneurship? Development and initial application of the SIE questionnaire. Educación y Entrenamiento. Emerald Group Publishing, Vol. 60 No. 7/8, pp. 800818. Recuperado de https://pdfs.semanticscholar.org/4a95/08631735dc08769a2c6881342b715e49cb28.pdf

Pontificia Universidad Católica del Perú. (Jueves 18 de octubre de 2018). Estudios para repensar la deserción. Lima: PUCP. Obtenido de Noticias y eventos: https://vicerrectorado.pucp.edu.pe/academico/noticias/estudios-repensar-la-desercion/ 
Sanchez, J., Ward, A., Hernández, B., \& \& Florez, J. (2017). Entrepreneurship education: state of the art. Propósitos y representaciones, 5(2), 401-473. Recuperado de https://files.eric.ed.gov/fulltext/EJ1159405.pdf.

Soto, J. L., \& Torres, C. A. (2016). Percepciones y expectativas del aprendizaje en jóvenes universitarios. Revista de Docencia Universitaria REDU, 14(1), 51-68. Recuperado de Recuperado de https://polipapers.upv.es/index.php/REDU/article/view/5797/6350.

Strauss, V. (13 de June de 2014). One thing we can learn from Shanghai: How to develop teachers. The Washington Post, págs. Recuperado de https://www.washingtonpost.com/news/answer-sheet/wp/2014/06/13/one-thing-wecan-learn-from-shanghai-how-to-develop-teachers/.

Vara, A. (2012). Desde la idea hasta la sustentación: 7 pasos para una tesis exitosa. Un método efectivo para las ciencias empresariales. Lima : Instituto de Investigación Universidad San Martìn de Porres. Recuperado de http://www.administracion.usmp.edu.pe/wpcontent/uploads/sites/9/2014/02/Manual_7pasos_aristidesvara1. 